\title{
EFFECT OF ROBOT-ASSISTED AND UNASSISTED EXERCISE ON FUNCTIONAL REACHING IN CHRONIC HEMIPARESIS
}

\author{
L. E. Kahn ${ }^{1,2}$, M. L. Zygman ${ }^{1}$, W. Z. Rymer ${ }^{1,2}$, D. J. Reinkensmeyer ${ }^{1,3}$ \\ ${ }^{1}$ Sensory Motor Performance Program, Rehabilitation Institute of Chicago, Chicago, IL, USA \\ ${ }^{2}$ Department of Biomedical Engineering, Northwestern University, Evanston, IL, USA \\ ${ }^{3}$ Department of Mechanical and Aerospace Engineering, University of California - Irvine, Irvine, CA, USA
}

Abstract - A common therapeutic approach for the rehabilitation of patients with hemiparesis involves repetitive voluntary movements with manual assistance from a therapist ("active-assist therapy"). We used a novel robotic device to deliver a controlled form of active-assist therapy in chronic stroke patients $(N=7)$. To examine the utility of direct mechanical assistance in rehabilitation of voluntary arm movements, a matched group of subjects with chronic hemiparesis $(N=7)$ performed the same repetitive exercises without the aid of the robotic device. Each group performed 24 therapy sessions over 8 weeks. We found that both groups demonstrated significant improvements in straightness of voluntary reaching movements, with limited improvements in range. Only the group that received robotic therapy significantly improved the smoothness of reaching. Improvements in both groups transferred to an unpracticed reaching movement and the timed performance of functional tasks. There were no significant differences in the magnitude of improvements between the two groups. These results suggest that it is the action of repetitively attempting to move, rather than the mechanical assistance provided by the robot, that stimulates arm movement recovery. However, imposing a smooth trajectory during practice of the reaching movements may help subjects learn how to produce smoother movements. In addition, practicing robot-assisted or unassisted reaching movements apparently improves control processes that generalize to other functional movements.

\section{INTRODUCTION}

Vascular injury in the brain often results in significant impairment to voluntary movement. This impairment usually includes deficits in strength (hemiparesis) and coordination as well as hyperexcitablity of reflex pathways. Voluntary repetitive movements have been shown to enhance the recovery of upper extremity movement in hemiparesis after brain lesion [1-3]. Recently, voluntary repetitive exercises administered with the mechanical assistance of robotic "rehabilitators" have also proven effective in improving arm movement ability in both acute $[4,5]$ and chronic $[6,7]$ populations after stroke.

Although repetitive movement with and without robotic assistance have both been shown to be of value, the role of the mechanical assistance in stimulating recovery remains unclear. Additionally, it remains unclear to what extent recovery remains specific to the therapy task, and to what extent robotic and non-robotic exercise aid the functional recovery of neuromuscular control of movement.

We aimed to address these two issues using a novel robotic device, the Assisted Rehabilitation and Measurement Guide (ARM Guide) [7], to provide assistive therapy to

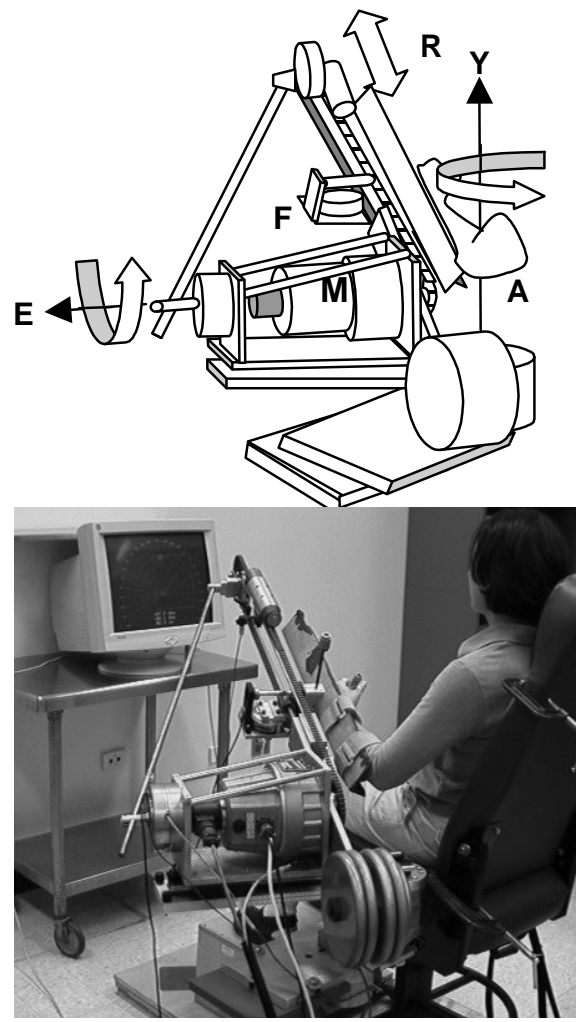

Fig. 1. Diagram and photo of the ARM Guide

patients with chronic hemiparesis. Using the device, subjects reached towards a series of targets in their workspace and their movements were constrained to follow a "normal" prescribed trajectory. For comparison another group of subjects performed a matched amount of exercise without any guidance from a device. This paper presents the effects on functional arm movement from fourteen subjects.

\section{METHODS}

\section{A. Robotic Device}

The ARM Guide (Fig. 1) has three controlled degrees-offreedom. A DC servo motor (M) controls the position of the subject's arm (A), which is coupled to the device through a trough and handpiece, along a linear track in the reaching direction (R). The other two degrees of freedom about the yaw (Y) and elevation (E) axes are controlled by magnetic particle brakes coupled to elastic components which provide a fixed compliance in these directions. Optical encoders record the positions of the device in the reaching direction and around the yaw and elevation axes. The handpiece slides along a linear track in the reaching direction (R) 
with a six-axis load cell $(\mathrm{F})$ reporting the forces and torques at the interface between the subject and the device. The device is statically counterbalanced so that it does not gravitationally load the arm.

\section{B. Subjects and Protocol}

Fourteen subjects with chronic hemiparesis (at least 6 months post-insult) were assigned to one of two exercise protocols. Subjects were matched by their impairment level, as assessed using the Chedoke-McMaster Evaluation of the Upper Extremity [8]. The first group, termed the "robot group" ( $\mathrm{N}=7)$, participated in twenty-four exercise sessions that lasted approximately one hour each over the course of eight weeks. During each session the subject performed reaching movements with the ARM Guide towards five different targets throughout the workspace (targets 2-6 in Fig. 2). The device was programmed to move using a proportional-derivative position controller along a minimumjerk trajectory with a velocity specified by the investigator. If the subject was unable to complete the reach or moved too slowly the device would assist through the end of the reach, with the firmness of assistance increasing exponentially throughout the movement [9]. The device would resist movement when a subject had a higher amount of function and tried to reach too fast. Real-time visual feedback of the location of the arm along the track, the yaw and elevation angles of the track, and the target location was given on a monitor in front of the subject (Fig. 1).

The second group, or "free-reaching group" $(\mathrm{N}=7)$, performed a matched amount of non-robotic exercise. Subjects sat in front of a black screen with numbered targets that were identically located to the targets used with the ARM Guide. The subject was instructed to reach as far as he or she could towards the target at a comfortable pace. No mechanical assistance or constraint was provided to the movement. A Flock of Birds electromagnetic motion analysis system (Ascension Technologies, Inc.) was used to record the end-point (hand) motion throughout the reach. Care was taken

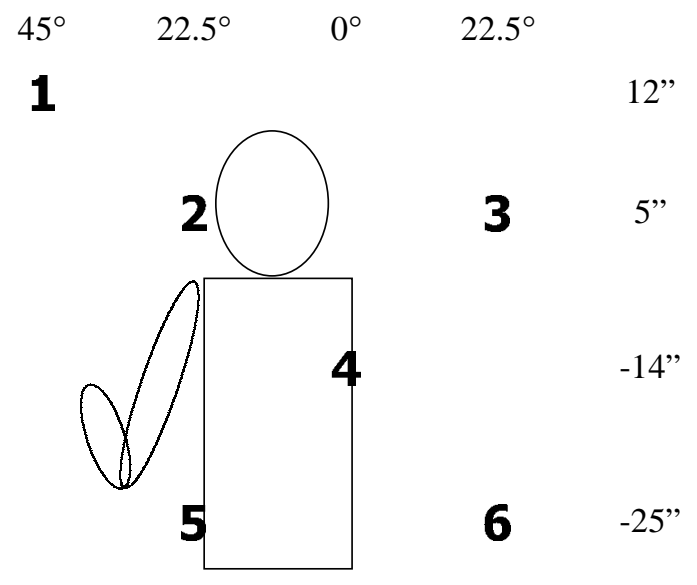

Fig. 2. Target locations for both the robot and free reaching exercises shown for the left arm. The target system is centered ( $0^{\circ}$ and 0 ") at the center of the subject's shoulder. to remove ferrous metals from the workspace of the sensor, and the Flock of Birds readings were tested to be accurate to at least $1 \mathrm{~cm}$. Trunk movement was minimized by fastening a harness around the shoulders and chest.

A key outcome measure involved subjects participating in a functional assessment to quantify voluntary arm movements. During a single assessment, subjects performed eight unassisted reaches to all six targets shown in Figure 2. To test for a possible transfer of improvements to unpracticed workspace regions, target 1 (Fig. 2) was included in the assessment even though there was no training to that target. To quantify each subject's ability to perform functional tasks the Functional Test of the Upper Extremity was administered by a blinded therapist. In this test, the time to complete simple daily tasks (e.g. pillow into a pillowcase, shirt tuck, etc.) was recorded. This test has been shown to have high inter- and intra-rater reliability [10]. Each subject participated in three evaluations with the Flock of Birds and one Functional Test both before and after the therapy program.

\section{Analysis}

The hand trajectories recorded by the electromagnetic tracking system were analyzed to examine the quality of each subject's movement. Specifically, the range, smoothness, and straightness of each reach were evaluated [11]. Range was quantified as the maximum distance moved away from the reach start position (i.e. hand in lap).

Smoothness of a movement was quantified as the number of peaks in the tangential speed signal for each reach. To calculate the number of speed peaks, the norm of the tangential speed from the start to the end reach point was scanned for local maxima and minima. A peak was counted if the increase from a minimum to the next maximum was above $15 \%$ of the global maximum speed in the reach.

Patients with hemiparesis commonly do not move in a straight path during a point-to-point movement. To quantify the straightness of movement, the length of the path followed by the hand from the starting point to the end reach point was calculated. The straightness of the reach was then quantified as the percent distance traveled along this path beyond the length of a straight line between the start and end reach points. For example, a straightness score of 50\% corresponded to traveling 50\% farther than a straightline path.

To test for an effect of therapy, these reach measures were calculated for the three pre- and three post-therapy evaluation sessions. A single score was derived for each subject for each session, and the average of this score for the three pre- (or post-) therapy evaluations was calculated, yielding a single pre- (or post-) score for each subject. Pre- to post- differences were evaluated for each group using one-sided, paired t-tests. Scores for the robot and free reaching groups were compared using t-tests.

\section{RESULTS}

No significant changes in reaching range were observed for either group (Fig. 3) following therapy. However, the mean range across subjects to the targets that were practiced (2-6) showed slight improvement, with the robot group approaching significance. The 

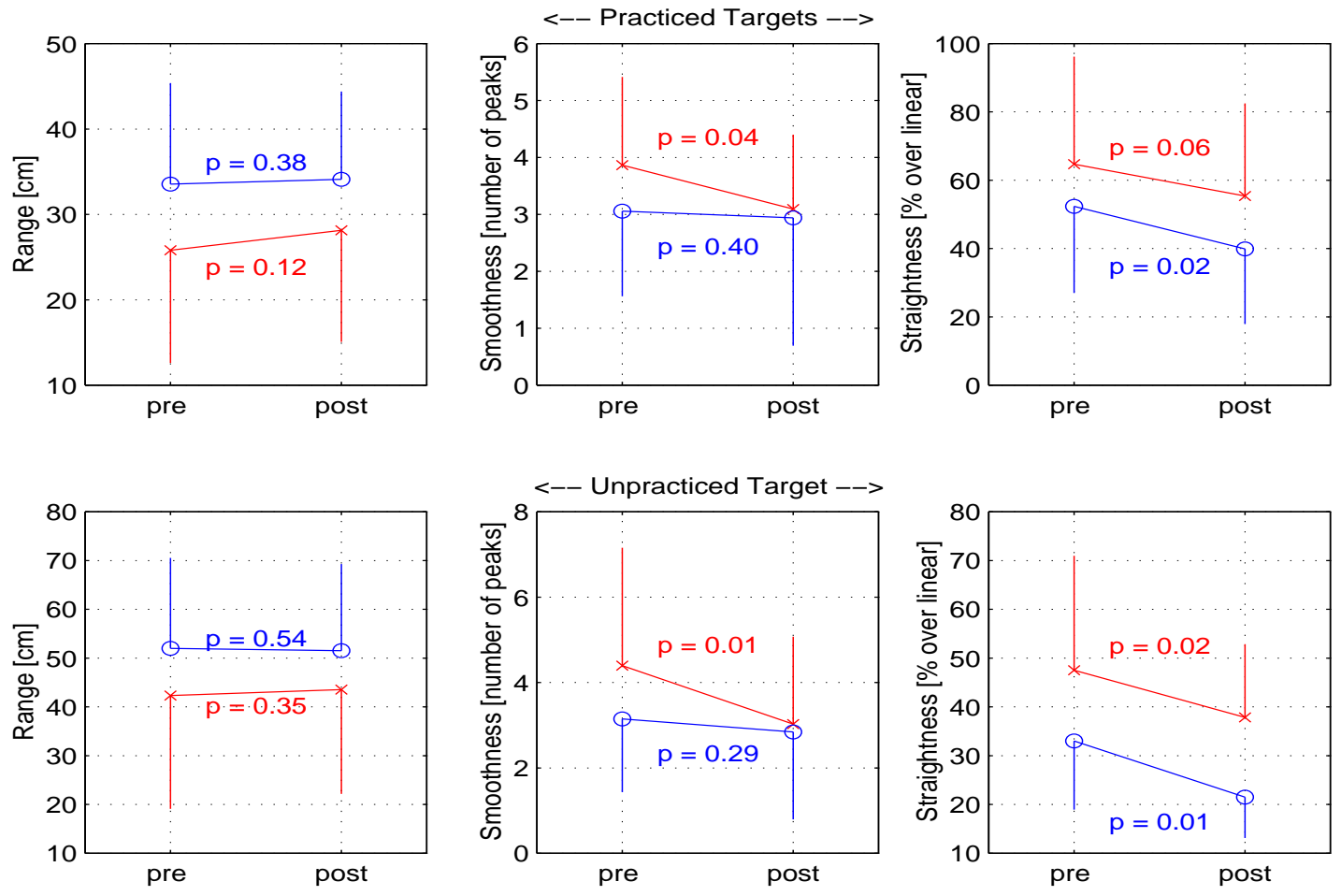

Fig. 3. Top Row: The change in range, smoothness, and straightness after 8 weeks of robotic (x) or free-reaching (o) therapy across all subjects in each group and across all targets used in training ( \pm s.d.). Bottom row: the same measures for the one target that was outside the training region.

means to the unpracticed target (1) did not significantly improve with practice for either group.

The robot group significantly improved the smoothness of their movements, while the free reaching group did not. Improvements in smoothness were also seen for the robot group for the unpracticed target.

Both the robot and free reaching groups significantly improved the straightness of their movements with training. These improvements again transferred to the unpracticed target.

Both groups improved in their ability to complete a series of functional tasks (Figure 4), quantified as a decrease in the time taken to complete the tasks. The decrease for the free reaching group was statistically significant, while the decrease for the robot group approached significance. No significant differences were observed between robot and free reaching groups in pre-, post-, or pre-post improvement for any of the measures.

\section{DISCUSSION}

The results of this study are relevant to two key questions in stroke rehabilitation.

A. What is the role of direct mechanical assistance in the rehabilitation of voluntary arm movement?

Both the robot and free reaching groups significantly improved arm movement ability. However, there were no significant differences between the groups, as quantified by the kinematic and clinical measures of arm movement. As reported previously, we also found no significant differences between the robot and free reaching groups using biomechanical measures of range, velocity, and tone acquired with the ARM Guide [12]. As both forms of exercise incorporate repetitive voluntary motion as an essential element, we hypothesize that is the action of repetitively attempting to move, rather than the mechanical assistance provided by the robot, that is the primary stimulus to arm movement recovery. Such a hypothesis is consistent with other repetitive movement exercise paradigms that have been demonstrated to improve upper extremity movement ability following brain injury [1-3], and may also explain other robotic therapy results $[4,6]$.

Although the overall arm recovery was comparable between the two therapy techniques, the data did suggest a possible subtle difference. Specifically, the ARM Guide group was marginally more effective in improving the smoothness of their movements with therapy. The mechanical assistance provided by the ARM Guide was designed to generate smooth hand trajectories similar to normal point-to-point movements in healthy subjects, which features a single peak in the tangential speed. Imposing a smooth trajectory during practice of the reaching movements may help subjects learn how to reduce the number of speed peaks to produce smoother movements. 


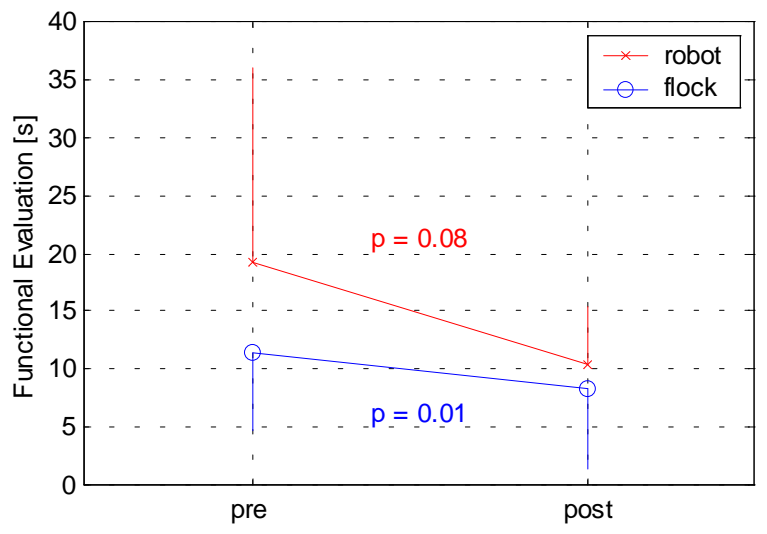

Fig. 4. The decrease in mean time needed to complete the tasks in the Functional Test of the Upper Extremity ( \pm s.d.)

\section{B. Does repetitive practice of one set of movements transfer to other movements?}

This question was addressed in two ways. First, movement ability was tested for a target to which the subjects did not practice reaching during therapy. While the improvement in range was still limited to this target, the improvements in smoothness and straightness were comparable to those for the trained targets. The transfer of training to other areas of the workspace suggests an improvement of the general control processes for reaching rather than refinement of motor programs specific to the trained targets.

Second, transfer to the performance of activities of daily living was evaluated. These activities were not explicitly practiced as part of therapy, and yet significant decreases in the times to perform these activities were observed. These results again suggest that practicing robot-assisted or unassisted reaching movements improves control processes that generalize to other movements.

\section{ACKNOWLEDGMENTS}

This research was supported in part by NIDRR FieldInitiated Grant H133G80052 and Whitaker Foundation Biomedical Engineering Research Grant to DJR and a NIH NICHD Institutional NRSA training grant to WZR.

\section{REFERENCES}

[1] Butefisch, C., Hummelsheim, H., Denzler, P. and Mauritz, K.H. "Repetitive training of isolated movements improves the outcome of motor rehabilitation of the centrally paretic hand." Journal of the Neurological Sciences, 1995. 130(1): p. 59-68.

[2] Liepert, J., Bauder, H., Wolfgang, H.R., Miltner, W.H., Taub, E., and Weiller, C. "Treatment-induced cortical reorganization after stroke in humans." Stroke, 2000. 31(6): p. 1210-6.
[3] Nudo, R.J., Wise, B.M., SiFuentes, F. and Milliken, G.W. "Neural substrates for the effects of rehabilitative training on motor recovery after ischemic infarct." Science, 1996. 272: p. 1791-1794. [4] Aisen, M.L., Krebs, H.I., Hogan, N., McDowell, F. and Volpe, B.T. "The effect of robot-assisted therapy and rehabilitative training on motor recovery following stroke." Archives of Neurology, 1997. 54(4): p. 443-6.

[5] Volpe, B.T., Krebs, H.I., Hogan, N., Edelstein, L., Diels, C., and Aisen, M. "A novel approach to stroke rehabilitation: robot-aided sensorimotor stimulation." Neurology, 2000. 54(10): p. 1938-44.

[6] Burgar, C.G., Lum, P.S., Shor, P.C. and Van der Loos, H.F.M. "Development of robots for rehabilitation therapy: The Palo Alto VA/Stanford experience." Journal of Rehabilitation Research and Development, 2000. 37(6): p. 663-673.

[7] Reinkensmeyer, D.J., Kahn, L.E., Averbuch, M., McKennaCole, A., Schmit, B.D., and Rymer, W.Z. "Understanding and treating arm movement impairment after chronic brain injury: Progress with the ARM Guide." Journal of Rehabilitation Research and Development, 2000. 37(6): p. 653-662.

[8] Gowland, C., Stratford, P. and Ward, M. "Measuring physical impairment and disability with the Chedoke-McMaster Stroke Assessment." Stroke, 1993. 24(1): p. 58-63.

[9] Reinkensmeyer, D.J., Takahashi, C.D., Timoszyk, W.K., Reinkensmeyer, A.N. and Kahn, L.E. "Design of robot assistance for arm movement therapy following stroke." Advanced robotics, 2000. 14(7): p. 625-637.

[10] Wilson, D.J., Baker, L.L. and Craddock, J.A. "Functional test for the hemiparetic upper extremity." American Journal of Occupational Therapy, 1984. 38(3): p. 159-64.

[11] Kamper, D.G., McKenna-Cole, A.N., Kahn, L.E. and Reinkensmeyer, D.J. "Alterations in reaching after stroke and their relationship to movement direction and impairment severity." Archives of Physical Medicine and Rehabilitation, 2001. in press.

[12] Kahn, L.E., Averbuch, M., Rymer, W.Z. and Reinkensmeyer, D.J., Comparison of robot-assisted reaching to free reaching in promoting recovery from chronic stroke, in Integration of Assistive Technology in the Information Age, M. Mokhtari, Editor. 2001, IOS Press: Amsterdam. p. 39-44. 